Oliveros, D., y Pérez, S. (2013). Medición de la competitividad de los productores de cacao en una región de Santander, Colombia. Revista Lebret (5). Bucaramanga, Colombia: Universidad Santo Tomás, pp 243-267

\title{
Medición de la competitividad de los productores de cacao en una región de Santander - Colombia*
}

\section{Measurement the Competitiveness of Cocoa Farmers in Santander, Colombia}

\section{Resumen}

Este trabajo evalúa la competitividad de los productores de cacao en Santander Colombia, mediante el modelo de las cinco fuerzas de Porter (2008), para ello se estudiaron los productores de la región de San Vicente del Chucurí y el Carmen de Chucurí (Santander-Colombia). Los resultados evidencian que la producción de cacao en grano durante los últimos años se ha convertido en una actividad productiva poco atractiva para la inversión debido a que los grandes márgenes de rentabilidad se los están quedando las empresas transformadoras de la cadena productiva. Asimismo, los cacaoteros de la región enfrentan una fuerte crisis financiera debido a la falta de productividad y competitividad en la producción del cacao en grano.

\section{Palabras clave}

Competitividad, Productividad, Productores de cacao

\section{Códigos de clasificación JEL: M1, Q1}

\begin{abstract}
This paper assesses the competitiveness of cocoa farmers in Santander Colombia, using Porter's five forces model; for this purpose, the producers of the region of San Vicente del Carmen Chucurí and Chucurí (Santander, Colombia) were studied. The results show that the production of cocoa beans in recent years has become an unattractive investment for the productive activity because the large profit margins are running for the processors of the production chain. Moreover the cocoa farmers in the region face a major financial crisis due to the lack of productivity and competitiveness in the production of cocoa beans.
\end{abstract}

\section{Keywords}

Competitiveness, Productivity, Cocoa farmers

* Artículo derivado del Proyecto de Invetigación " Análisis de la competitividad del sector, elaboración de cacao, chocolate y productos de confitería" financiado por la Universidad Autónoma de Bucaramanga (UNAB), Colombia.

1 Doctora en Sistemas Flexibles de Dirección de Empresas, Universidad Pública de Navarra. Docente Investigadora Universidad Autónoma de Bucaramanga-Colombia. Correo electrónico: doliveros364@unab.edu.co

2 Magister Gestión de la Empresa Multinacional, Universidad de Zaragoza. Docente Investigadora Universidad Autónoma de Bucaramanga-Colombia. Correo electrónico: sperez@unab.edu.co 


\section{Introducción}

El cacao es un alimento altamente nutritivo y un producto commoditie $e^{3}$ que se posiciona en el tercer lugar después del azúcar y el café en el mercado mundial. El mercado mundial del cacao en grano ha reflejado un visible desempeño en los últimos años, debido a una creciente dinámica de la demanda en los mercados tradicionales de Europa y Estados Unidos así como en los mercados de los países asiáticos. Es demandado principalmente por empresas transformadoras, los bienes intermedios que se derivan del cacao se emplean como materias primas no solo en la industria de chocolates, sino también en la industria de cosmética y farmacéutica, es decir, del cacao se pueden derivar varios productos, como por ejemplo, el chocolate, productos de confitería, la manteca de cacao, el licor de cacao, el cacao en polvo, la pasta de cacao, el chocolate de mesa, los perfumes, las cremas, los labiales, algunas medicinas, entre otros. Algunos de estos derivados son considerados bienes de lujo.

Es por esta razón que aunque los países en desarrollo son los principales productores de cacao, son los países desarrollados quienes principalmente consumen (demandan) el grano a nivel mundial, Esta tendencia creciente de la demanda ha dinamizado los precios y, en consecuencia, ha estimulado la oferta de la materia prima $^{4}$.

Colombia es uno de los países que mayor producción de cacao genera en América después de Brasil, Ecuador, Perú y República Dominicana. Según la Organización Internacional del Cacao (ICCO) $)^{5}$. Colombia produce uno de los mejores cacaos del mundo por su aroma y sabor, distinción que solo tiene el 5\% del grano mundial.

El cultivo de cacao se encuentra presente en casi todas las regiones de Colombia y el Departamento que tradicionalmente ha concentrado la mayor producción de cacao es Santander, que para el 2011 aportó cerca del 38\% de la producción nacional (ICCO, 2007)

En Colombia en los últimos años, el gobierno ha venido desarrollando una política agrícola y programas de desarrollo alternativo que se han encaminado a la promoción

3 Los commodities o productos primarios constituyen la materia prima para obtener bienes intermedio y de consumo final por parte de los consumidores.

4 Para mayor información revisar el documento elaborado por el equipo Economic Research Service-ERSUSAID/ MIDAS Crops, escrito por María del Pilar Ruiz Molina, Hernán Montoya Ortíz y María Alejandra Urueña del Valle, con la colaboración y los comentarios de Carlos Federico Espinal Gómez y Roberto Albornoz Miranda.

5 La ICCO fue creada en 1973 bajo el auspicio de las Naciones Unidas para administrar las disposiciones del Convenio Internacional del Cacao de 1972 y de los Convenios posteriores de 1975, 1980, 1986, 1993 y 2001. Es un organismo al que pertenecen la gran mayoría de los países productores y consumidores de cacao, siendo uno de sus objetivos principales el fomento y la colaboración internacional e interinstitucional en temas relacionados con el cacao, actuando como escenario de alcance mundial entre productores, consumidores e industriales, a fin de contribuir a la estabilización del mercado y al abastecimiento asegurado a precios razonables, promoviendo la transparencia del mercado de cacao y fomentando la investigación científica y el desarrollo en temas relacionados con la producción, la transformación y el consumo de cacao; se resalta que aún Colombia no hace parte de esta organización (ICCO, 2006). 
y difusión de aquellos cultivos para los cuales el país presenta ventajas comparativas a nivel internacional. Así, en el país este es uno de los cultivos permanentes que ha logrado una mayor dinámica y expectativa en materia de establecimiento de nuevas áreas, debido a las condiciones edafoclimáticas con las que se cuenta. Según un estudio de Corpoica y Fedecacao (2009) Colombia cuenta con una potencialidad de dos millones de hectáreas aptas para el desarrollo de cultivos de cacao y para el gobierno busca que se produzcan 246.000 toneladas de cacao.

El cultivo del cacao forma parte del primero de tres eslabones que componen la cadena de cacao en el país. Los otros dos los constituyen el eslabón de la comercialización y el eslabón industrial. Según el Observatorio Agrocadenas Colombia, el eslabón primario o de producción abarca los procesos de siembra, mantenimiento y recolección de cacao, donde se encuentran todos los agricultores o dueños de las tierras y productores de insumos. El eslabón secundario comprende la comercialización del grano, tanto a nivel interno como externo, desde el momento en que el grano es comprado por los agentes, hasta que es colocado en la puerta de las fábricas procesadoras o en el país de destino de las exportaciones y finalmente, el eslabón terciario es el industrial que abarca el procesamiento del grano para producir bienes intermedios.

De acuerdo con estimativos del Observatorio de Agrocadenas, se calcula que el valor agregado por el eslabón primario de la cadena (cacao en grano) en Colombia representa tan sólo el 15\% del valor bruto total de la cadena, concentrándose la mayor cantidad de valor en el eslabón industrial (Espinal, Martínez y Beltrán, 2005).

Debido a esto, existe una problemática generalizada entre los productores de cacao en Colombia que está rebozando los límites, según los productores el lado amargo del chocolate está antes de su elaboración y el precio se convierte en su mayor preocupación ${ }^{6}$, los productores son los que menos beneficios están alcanzando en la cadena de cacao $^{7} \mathrm{y}$, ante esta problemática, es necesaria la búsqueda de soluciones adecuadas que permitan mejorar la competitividad y en especial, plantear alternativas que busquen mejorar las condiciones de los productores, y el rendimiento productivo y económico de este sector.

$6 \quad$ El cacao al estar categorizado como productos básicos o commodities que se caracterizan por tener un bajo grado de transformación, lo hace un producto relativamente homogéneos, razón por lo cual se pueden transar de manera masiva en los mercados internacionales a un precio único. En este contexto, los precios de este tipo de bienes dependen en buena medida de los inventarios disponibles en los principales mercados en que se transan.

7 http://www.revistaialimentos.com.co/ediciones/edicion-10/sector-destacado-confiteria-y-chocolateria/elcacao-de-colombia-busca-consolidarse.htm 


\section{El cacao en el mundo}

La situación de la oferta y demanda de cacao nivel mundial se ha caracterizado en general por amplias fluctuaciones. Por ejemplo, para el período desde 2002 a 2012 -según estimaciones de la secretaria ICCO - la demanda excedió la oferta (ICCO, 2012), por lo que puede afirmarse que existe un entorno favorable para la actividad productiva.

Según la Organización Internacional para el Cacao (ICCO) (2012), la producción de cacao en el mundo durante las últimas cinco temporadas ha estado concentrada en el continente Africano, con un porcentaje que oscila entre el $68 \%$ y $75 \%$, en segundo lugar se han mantenido los países asiáticos y Oceanía y finalmente, los países americanos con una participación entre el 12\% y $14 \%$.

Los países productores del África son: Costa de Marfil, Ghana, Nigeria, Togo y Camerún, los cuales concentran una producción promedio del 98\% (véase Figura 1); el primer lugar lo ocupa Costa de Marfil con una producción promedio del $49 \%$ y en segundo lugar esta Ghana con una producción promedio del 28\% (Asamoah y Annan, 2012).

Figura 1. Productores de cacao en África (millones de toneladas)

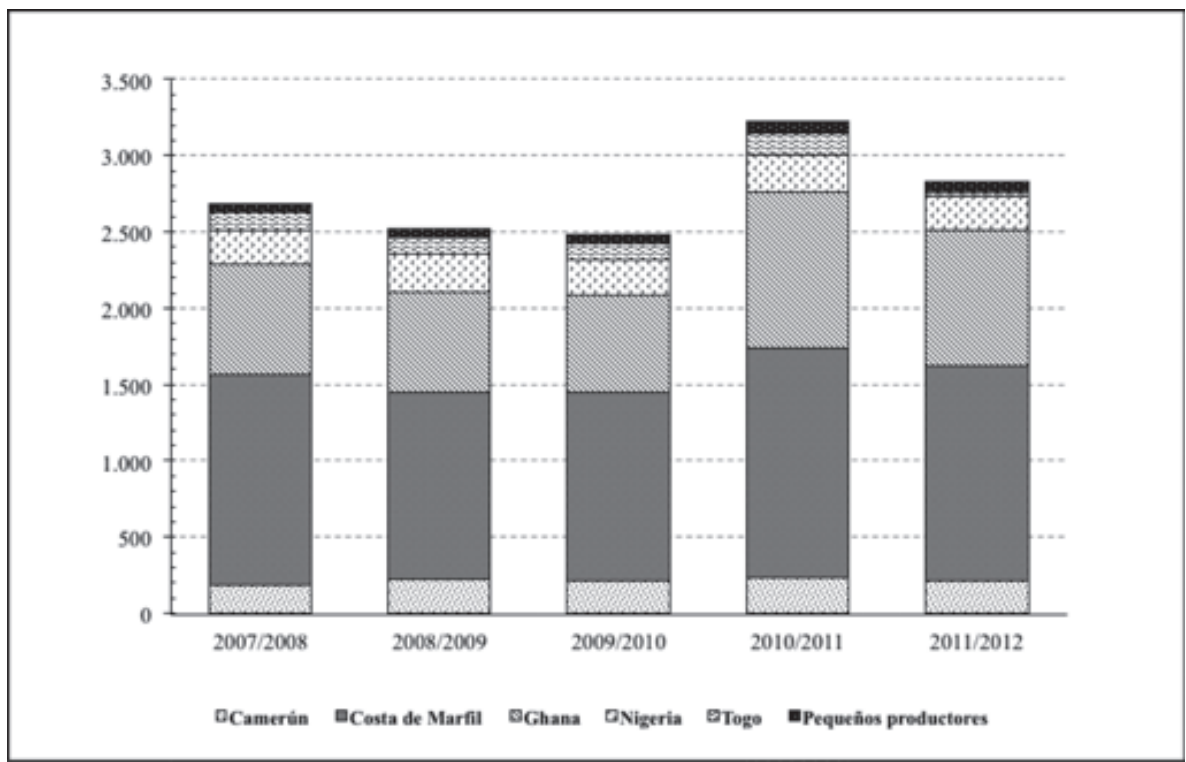

Fuente: Autoras a partir de International Cocoa Organization - ICCO (2012)

"The Word cocoa economy: past an present". 
En América, los mayores productores de cacao durante el período 2011-2012, según la cantidad de producción expresada en millones de toneladas y según estimaciones de ICCO (2012 son: Brasil (190), Ecuador (175), Perú (50), República Dominicana (60) y Colombia (45). Brasil representa en promedio el 34\% de la producción; en segundo lugar está Ecuador con el 28\%; en tercer lugar República Dominicana con el 11\%; en cuarto lugar Perú con el 8\% y Colombia sube al 5 lugar con el 7\% (véase Figura 2). A pesar de ser países agrícolas, los países latinoamericanos no tienen acceso a la tecnología de producción masiva. Por el contrario, se estima que aproximadamente el 95\% de la producción anual procede de pequeños productores, con una superficie de sembrado de entre una y tres hectáreas (ICCO, 2007).

Figura 2. Producción de cacao en grano en América (millones de toneladas)

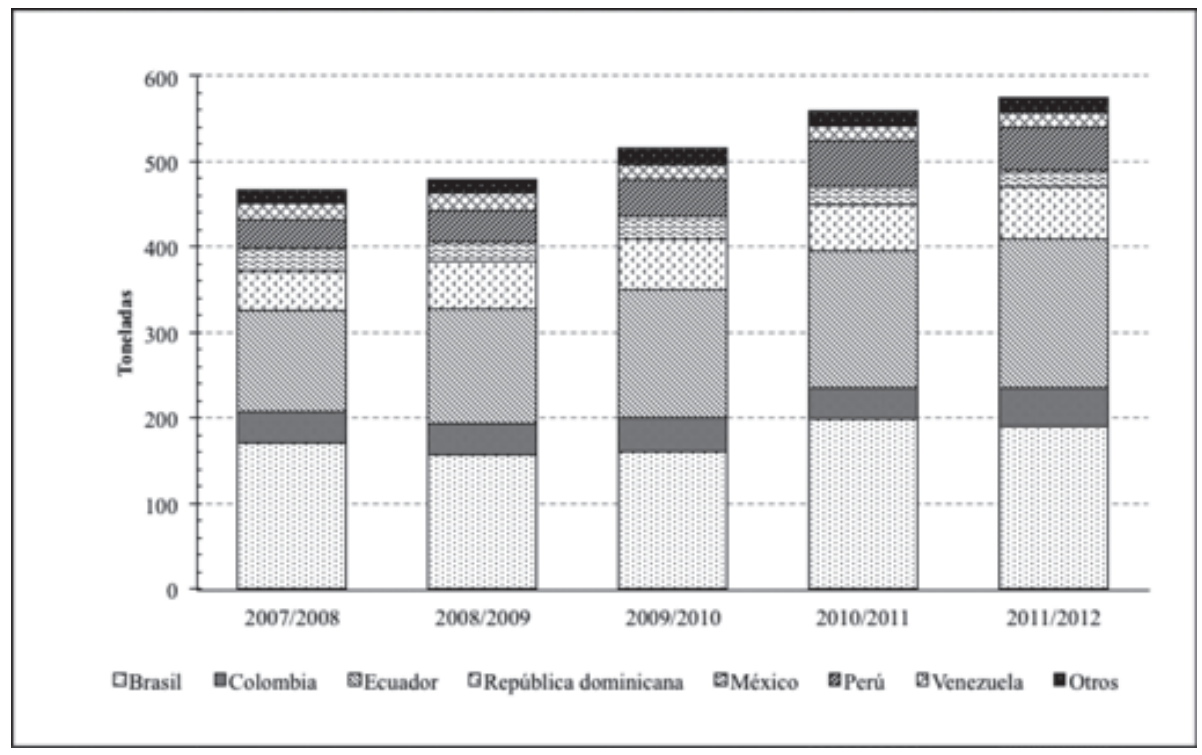

Fuente: Autoras a partir de International Cocoa Organization - ICCO (2012)

"The Word cocoa economy: past an present".

Durante los últimos años, la situación de la oferta y demanda de cacao se ha caracterizado en general por amplias fluctuaciones, mientras que la producción se ha incrementado de forma errática, con índices de crecimiento anual de entre el $-10 \%$ y el $+18 \%$, las moliendas han ido aumentando constantemente a un ritmo más lento de entre un 2\% y un 7\%. Así, la producción mundial de cacao se aumentó pasando de casi 3,2 millones de toneladas en la campaña cacaotera 2002-2003 a una cifra estimada de 4 millones de toneladas a 2012, durante la campaña 2010-2011, se 
alcanzó una cifra récord de producción, que superó los 4,3 millones de toneladas, gracias a las excelentes condiciones climáticas que favorecieron el desarrollo de la cosecha por toda África, la mayor región productora de cacao a nivel mundial.

Diferentes proyecciones para el cultivo del cacao argumentan que América aumentará su producción de un $15.7 \%$ a $16.1 \%$ en la próxima década y Asia y Oceanía, aumenten también su producción del 13\% al 14,1\%. Sin embargo, África disminuirá su producción de 71,8\% a 69,8\% aunque seguirá siendo la principal región productora mundial de cacao en el próximo decenio. Por otra parte, se prevén crecimientos del 1,8 por ciento y 0,5 por ciento de las producciones de la República Dominicana y México, respectivamente.

En Colombia, las proyecciones no son muy positivas. La utilización de nuevas variedades de clones que pueden aumentar la rentabilidad de la producción recientemente no llevaría la producción a los niveles alcanzados durante los años 1980, porque algunos productores, desalentados por los bajos precios mundiales registrados últimamente, han pasado a otros cultivos (FAO, 2004). No obstante, como se mencionó anteriormente, Colombia cuenta con una potencialidad de 2 millones de hectáreas aptas para el desarrollo de cultivos de cacao de las cuales aproximadamente 478.800 están ubicadas en Santander y 401.684 en Casanare.-

\subsection{Los precios del cacao}

Los precios internacionales del cacao como commodity se basan en la oferta y la demanda. Las cotizaciones se dan en la Bolsa de Londres y en el Mercado de Café, Azúcar y Cacao de Nueva York. Por tanto, cuando existen excedentes de producción los precios caen y se desestabiliza el mercado, provocando desaliento entre los productores que fácilmente migran a otras actividades. Cuando la producción disminuye debido a enfermedades fitosanitarias, fenómenos climáticos, problemas políticos y sobre todo problemas culturales, reaccionan positivamente los precios. Los precios diarios según la ICCO han oscilado entre US\$ 1.361 y US\$ 3.730 por tonelada entre los años 2002 y 2012. El precio mínimo se alcanzó en mayo de 2004, cuando el mercado experimentó un superávit masivo de producción de casi 290.000 toneladas y el precio máximo se alcanzó en la producción en 2010-2011 donde se registraron los precios más elevados en 30 años (véase Figura 3). Sin embargo, estos niveles máximos se imputaban principalmente a la prohibición de exportación de cacao de Côte d'Ivoire - el mayor productor de cacao - quien bajó abruptamente su producción por problemas políticos internos; por otro lado Ghana e Indonesia se han visto sumamente afectadas a través de los años por el clima, el envejecimiento de los árboles y las enfermedades; por lo que su producción descendió allí también significativamente (González, 2005; Ofori-Bah y Asafu-Adjaye, 2011).

Las fluctuaciones en los precios de la mayoría de los países productores, son el reflejo de la evolución de los precios internacionales, las variaciones del valor 
internacional de la moneda local, y la modificación de las estructuras y condiciones del mercado local específico, entre ellas la fiscalización, la competencia, la distancia del puerto y la calidad. Esta última se ha visto afectada por los precios bajos, que han impedido que los productores puedan fertilizar, contratar asistencia técnica y fumigar el campo, lo cual podría ayudar a mejorar las condiciones de las plantaciones (Figura 3).

Figura 3. Fluctuaciones en los precios US\$ tonelada Periodo 2000-2010

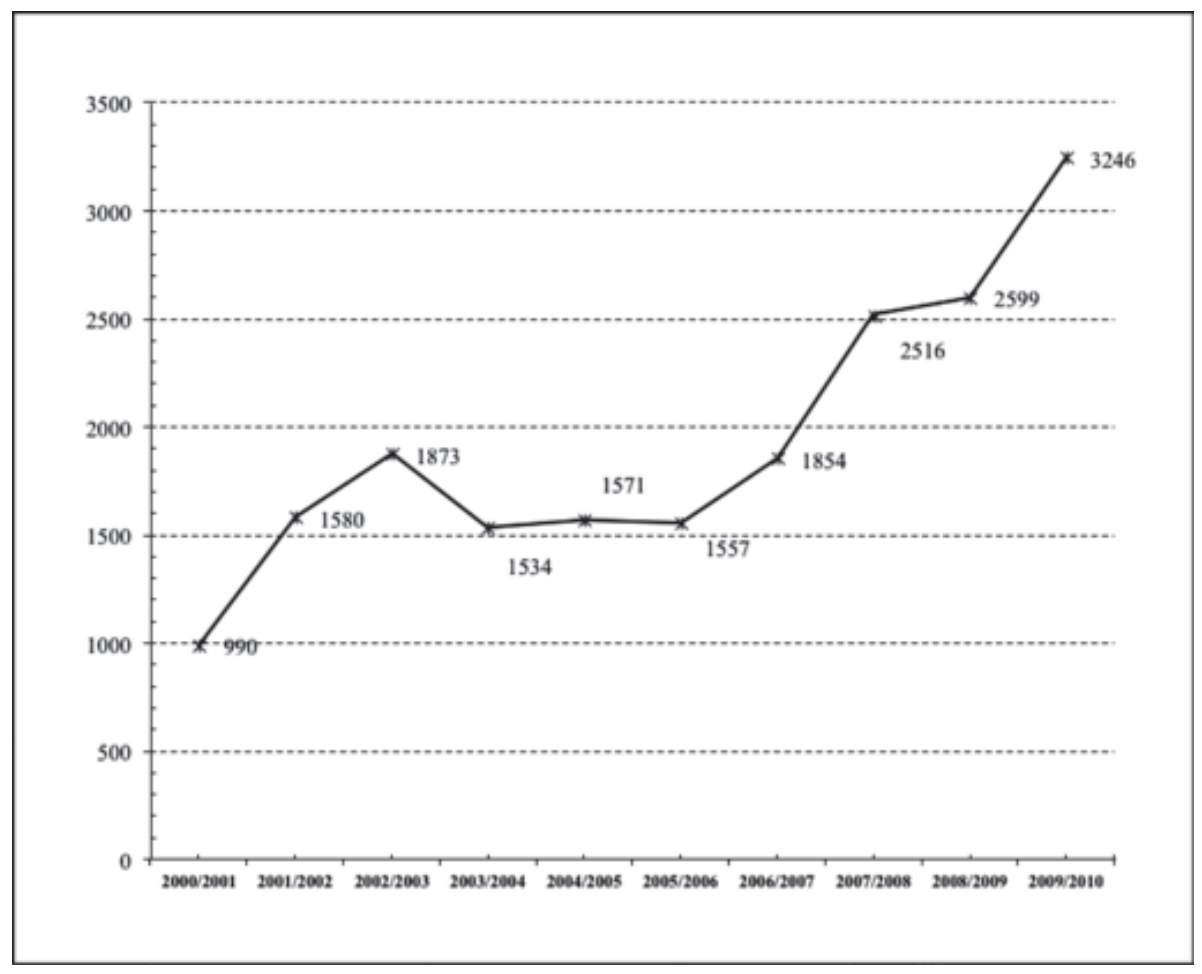

Fuente: Autoras a partir de International Cocoa Organization - ICCO (2012) "The Word cocoa economy: past an present".

\subsection{Consumidores de cacao en el mundo}

Las exportaciones de cacao seguirán siendo de cacao en grano, a pesar de un cierto aumento de la capacidad de elaboración de los países productores, especialmente de África. En el mercado internacional la producción de cacao se distribuye principalmente en América Latina, África y Asia. Sin embargo, la elaboración de productos derivados del cacao se realiza principalmente en Europa y en Estados 
Unidos (INFOCOMM, 2007). El consumo intermedio de cacao se encuentra concentrado en los países desarrollados, tanto para la elaboración de productos como de sub-productos. Empresas como Cargill, Archer Daniella, Midland, Blommers, Chocolate Company y World Finest Chocolate Company procesan subproductos, entendiendo por éstos: pastas, licor de cacao, manteca de cacao y polvillo. Mientras que empresas como: Nestlé, Mars Inc, Cadburry Scheweppes, Ferrero y Hershey's Food procesan productos terminados como chocolates y cocoas (González, 2005).

Según la Organización Internacional del Cacao ICCO (2012) el consumo de productos de confitería de chocolate aumentó entre los años $2002-2010$ un 10\% en países europeos, Estados Unidos, Brasil, Japón y Australia, tratándose de una tasa anual de crecimiento del 1,2\%. No obstante, en el año 2009 se registró un descenso en el consumo de chocolate en la mayoría de estos países debido a la fuerte crisis económica que atravesaban. Sin embargo, para el año 2010 el consumo empezó a reactivarse, aumentando en 2,8\%, para alcanzar un nivel récord de alrededor de 5,54 millones de toneladas ${ }^{8}$.

En general, se calcula que el consumo de productos derivados del cacao ha aumentado significativamente, creciendo a una tasa media del $2.5 \%$ anual en la última década. Si persiste esta tendencia, la demanda de cacao se duplicará cada 25 o 30 años. Mientras que un europeo consume dos kilos de chocolate al año, los chinos consumen 100 gramos y la India 500 gramos. Si el gusto por el chocolate sigue creciendo y el consumo aumenta a un kilo por habitante asiático, la industria se vería incapacitada de satisfacer la demanda.

\section{El cacao en Colombia}

Un componente marginal del total de segmentos que componen la oferta agrícola colombiana es la producción de cacao en grano, según datos del Ministerio de Agricultura y Desarrollo Rural de Colombia (2006) en 2005 el cacao representó $0,8 \%$ del valor de la producción agrícola, $0,21 \%$ del volumen de esa producción y $2,8 \%$ del área agrícola total cultivada.

El cultivo de cacao se encuentra presente en casi todas las regiones de Colombia, sin embargo, como en la mayoría de los cultivos, existe una cierta concentración o regionalización del producto. El Departamento que tradicionalmente ha concentrado la mayor producción de cacao es Santander que para el año 2011 aportó cerca del 38\% de la producción nacional. Le siguen en importan cia Nariño, Huila, Norte de Santander, Antioquia, Tolima y Arauca, regiones que a futuro presentan amplias expectativas para difundir el cultivo de cacao, debido a sus condiciones edafoclimáticas (véase Tabla 1) . 
Medición de la competitividad de los productores de cacao en una región de Santander ...

Tabla 1. Producción de cacao en Colombia por Departamentos 2008-2011

\begin{tabular}{ccccc}
\hline Departamento & $\mathbf{2 0 0 8}$ & $\mathbf{2 0 0 9}$ & $\mathbf{2 0 1 0}$ & $\mathbf{2 0 1 1}$ * \\
\hline Santander & 47.646 & 48.044 & 49.429 & 53.929 \\
Nariño & 9.855 & 10.458 & 10.940 & 12.440 \\
Huila & 12.305 & 11.045 & 11.200 & 12.180 \\
\hline Norte de Santander & 9.541 & 9.978 & 10.190 & 11.390 \\
Antioquia & 7.608 & 8.045 & 8.290 & 10.870 \\
Tolima & 9.869 & 10.006 & 10.140 & 10.215 \\
Arauca & 8.064 & 8.304 & 8.460 & 8.510 \\
Otros & 19.141 & 22.108 & 22.991 & 24.111 \\
\hline Total & 124.029 & 127.988 & 131.640 & 143.645 \\
\hline
\end{tabular}

Fuente: Proexport (2012).

Según cifras de la Federación Nacional de Cacaoteros de Colombia (FEDECACAO) y el Ministerio de Agricultura y Desarrollo Rural (MADR) (2006) en el Departamento Santander se encuentra cerca de 50\% de las unidades productivas $\mathrm{y}$, por tanto, el cultivo de cacao tiene gran importancia social.

En el país existen aproximadamente 25.000 agricultores que siembran y recolectan. La producción de cacao genera cerca de 52.000 empleos, es decir, que representa $2,8 \%$ del empleo en cultivos permanentes en Colombia. No obstante, la mano de obra del cultivo tiende a ser en su mayoría de carácter familiar. La producción de cacao tiene la ventaja de ofrecer un ingreso garantizado a los cultivadores, puesto que la industria transformadora demanda la totalidad de la producción $\mathrm{y}$, generalmente, garantiza la compra total de la cosecha nacional (Gamboa, Zuluaga y Rubiano, 2007).

Informes complementarios sobre el tema indican que en Colombia la producción anual de cacao en grano se obtiene de la explotación de alrededor de 90.000 hectáreas sembradas en aproximadamente 25.000 fincas, con un rendimiento promedio por hectárea cosechada estimado entre 350- 450 kilos de cacao en grano, indicador que resulta muy bajo si se compara con otros países productores de cacao. Este bajo rendimiento en la producción es consecuencia principalmente de un deficiente manejo del cultivo, atribuible a la edad avanzada de las plantaciones sembradas, ya que los árboles de edad mayor a 20 años son más numerosos que los nuevos, en parte por el abandono de los cultivos en las épocas de bajos precios, que incidió en 
la propagación de plagas y enfermedades entre las que se destacan la Monilia ${ }^{9}$ y la Escoba de bruja ${ }^{10}$ al tipo de material de propagación utilizado (ej. cacaos híbridos y comunes con bajos niveles de tolerancia a plagas y enfermedades), a la baja densidad de árboles en producción por hectárea, al bajo nivel tecnológico de las fincas y a las dificultades para que el agricultor pueda poner en práctica las recomendaciones de manejo integral del cultivo entre otras factores.

Además, el cultivo de cacao enfrenta la complicación de contar con una deficiencia de capital humano (64\% de los agricultores tenían sólo el nivel primario de educación) que complica una mayor transferencia tecnológica (Gamboa et al., 2007). Ante esta realidad, cabe mencionar que el gobierno e instituciones como Corpoica y Fedecacao, y el sector privado ofrecen programas de transformación tecnológica que incluyen asistencia técnica, asesoría, suministro de semilla y material vegetativo clonado, rehabilitación y renovación de cultivos viejos entre otros.

\subsection{Precios del cacao en Colombia}

El precio es una señal fundamental para la decisión del productor agrícola de continuar cultivando un determinado producto. En el caso del cacao en Colombia productores enfrentan una estructura de demanda oligopsónica, pero el precio internacional tradicionalmente ha sido un referente importante para la determinación de precios del cacao en el mercado doméstico. Autores como Espinal et al. (2005) y Martínez, Espinal y Ortiz (2005) indican que los precios pagados al productor nunca han estado por debajo del precio de referencia que establece el Ministerio de Agricultura. El precio nacional pagado a los productores de cacao en Colombia también ha registrado notables fluctuaciones: así, ha pasado de pagarse a $\$ 2.245 /$ kilo en el año 2000, a \$3.701/kilo en el año 2005, \$4.332/kilo en el año 2008 para bajar a $\$ 3.762,5 /$ kilo a junio del 2013 . Esta inestabilidad ha incidido en que la producción crezca a un ritmo más lento, ya que en la actualidad los precios de venta no cubren los costos variables de producción y los cultivadores podrían verse obligados a abandonar sus cultivos.

\subsection{Comercialización del cacao en Colombia}

La comercialización del cacao y la absorción de la cosecha por parte de las empresas que conforman la industria de elaboración de productos derivados del cacao, se realiza mediante asociaciones de productores, comercializadores y exportadores. Cerca del $70 \%$ de la comercialización del grano se hace a través de comercializadores que se ubican en las cabeceras municipales y que, en ocasiones, reciben de la industria transformadora recursos para financiar la compra de cacao. La ganancia de estos comercializadores es la diferencia entre el precio al cual le

9 Enfermedad ocasionada por un hongo conocido como Moniliophthota roreri

10 Llamada así porque los síntomas iniciales son la deformación o desecación de los brotes tiernos de la planta 
compran al productor de cacao y el precio al que le venden a la industria nacional o a los exportadores (Martínez et al., 2005).

Se estima que el canal de los acopiadores representa $20 \%$ de la comercialización del cacao, estos agentes tienen una relación directa con el agricultor y en algunas ocasiones comercializan el cacao para los agentes comercializadores. Finalmente, existen unas pocas organizaciones de productores y cooperativas - que no son lo suficientemente grandes - que actúan como agentes comercializadores y canalizan $10 \%$ del intercambio del grano. Muchas de estas organizaciones han desaparecido debido a los bajos márgenes de comercialización y los bajos volúmenes de cacao disponibles y ante todo, por la competencia planteada por los comerciantes particulares que logran realizar el negocio a muy bajos costos en comparación con los que incurre la asociación, tales como seguros, administración, contabilidad, revisoría fiscal, entre otros (Martínez et al., 2005).

La producción Nacional de Cacao se concentra principalmente en dos empresas de transformación: Casa Luker y Nacional de Chocolates ${ }^{11}$ : entre el año 2004-2011 la participación de la compra de estas dos empresas fue aproximadamente el 84,5\%. Por tanto, se evidencia claramente las características de un mercado duopsónico, por la alta participación de los comercializadores y la elevada concentración de la compra final del cacao en muy pocas empresas. Esta situación conlleva a que las empresas líderes del sector posean cierto control sobre los precios y así, sobre sus competidores. No obstante, como se ha mencionado anteriormente, la fijación del precio nacional tiene como base el comportamiento del precio internacional.

La empresa Nacional de Chocolates presenta una posición dominante en la compra de cacao en los Departamentos de Santander y Antioquia, mientras que Casa Luker es quien posee mayor participación en los departamentos de Cundinamarca y Caldas. Las empresas medianas son Gironés, Chocolate Caldas y Tolimax y dentro de las pequeñas se destacan la Fábrica de Chocolates Andino, Compañía Occidental de Chocolates, J. Arbes Carrillo y Chocolates Santa Fé. Todas estas empresas compiten por el mercado de chocolate de mesa. Sin embargo, las empresas medianas y pequeñas presentan dificultades en la distribución del producto a nivel nacional, por lo que reducen su mercado a nivel regional y local y deben competir con las compañías líderes del mercado (Espinal et al. 2005).

\subsection{El cacao en Santander}

El Departamento de Santander se constituye en el más importante productor de cacao en Colombia y, aunque ha disminuido su cuantía en los últimos años, continúa siendo líder en esta actividad económica.

11 Que hoy forma parte del Grupo Nutresa S. A. 
Si bien en Santander existen varios municipios dedicados a esta actividad, el potencial de la misma se centra específicamente en los municipios de San Vicente y Carmen de Chucurí, cacaoteros por excelencia, los cuales cuentan con características edafo-climáticas especiales que favorecen la producción. Además de otros municipios como Landázuri, Rionegro y el Playón como los mayores exponentes de la producción y herencia de la calidad del cacao en Santander (Véase Figura 4). En las regiones mencionadas, el tamaño de las plantaciones se distribuye mayoritariamente en fincas de entre tres y diez hectáreas, siendo manejadas en su gran mayoría por sus propietarios $\mathrm{y}$, en menor medida, por arrendatarios.

Figura 4. Municipios productores de cacao en Santander

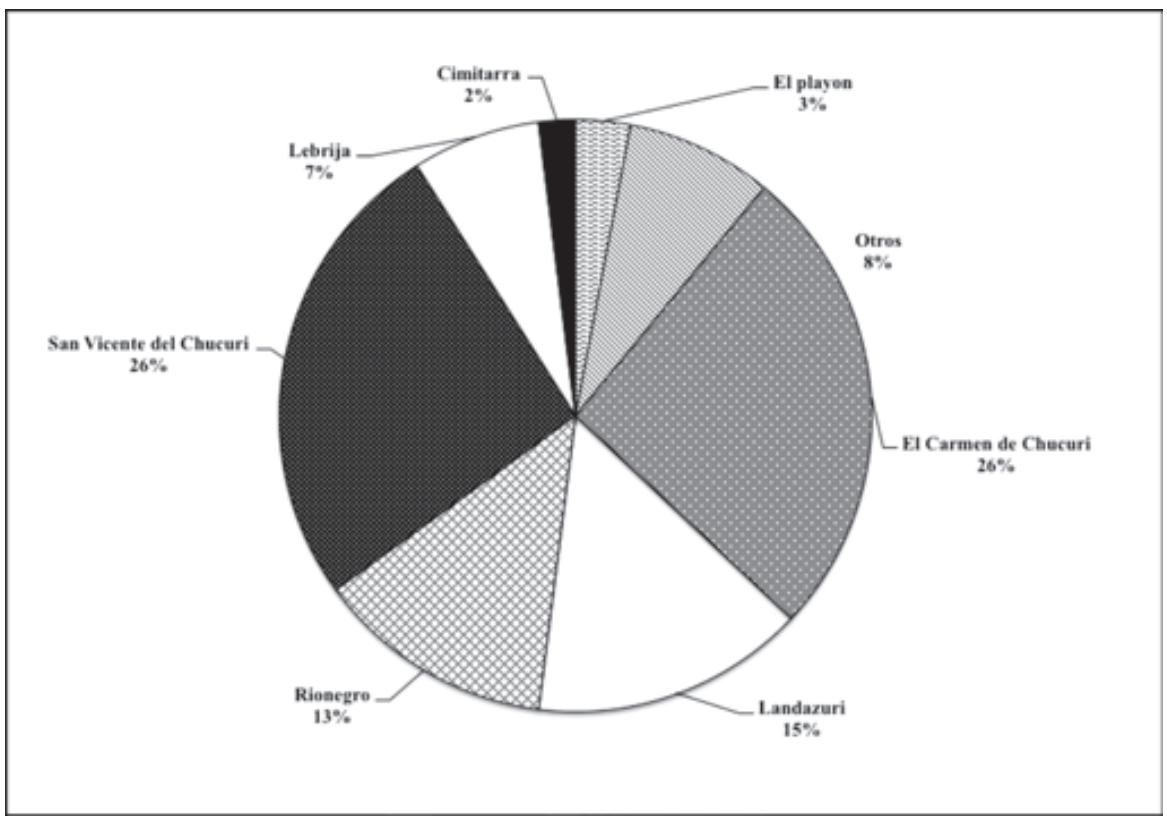

Fuente: Mojica, A y Paredes, J (2006).

La comercialización de cacao en Santander se efectúa a través de cooperativas y agricultores o personas naturales acopiadoras. Las cooperativas, además de participar en la comercialización del cacao en grano, propenden por garantizar a los consumidores que el producto sea de calidad y se constituyen en uno de los factores de regulación de precios al productor.

Las principales cooperativas cacaoteras en Santander se encuentran en los municipios de San Vicente de Chucurí, El Carmen de Chucurí, Landázuri, Rionegro, Bucaramanga, Cimitarra, El Playón, Lebrija, Santa Helena de Opón y la Belleza. 
Tan sólo en San Vicente de Chucurí y El Carmen de Chucurí se encontraron 16 cooperativas a las que pertenecen sólo el $30 \%$ de los productores.

Santander cuenta con buenos estándares de calidad y con el reconocimiento de la industria nacional. A pesar de ello, la industria regional posee una estructura artesanal, maquinaria obsoleta de baja eficiencia y tecnología que le impide ampliar su producción, por lo que ofrece básicamente chocolate de mesa a un mercado bastante limitado. Debido a la escasez de mano de obra, no se realiza adecuadamente el beneficio del grano y prefiere venderlo en estado húmedo, lo que incide en la calidad final, aroma y sabor, y por supuesto, en las ganancias obtenidas (Bueno, 2004). Estos aspectos plantean la necesidad de fortalecer prácticas en la cadena que promuevan la producción de un grano de mejor calidad (Castellanos Torres, Fonseca, Montañez y Sánchez,, 2007).

Para que Santander continúe manteniendo el liderazgo en producción de cacao es importante trazarse unas metas claras que satisfagan a todos los integrantes de la cadena productiva de cacao en especial a los productores del grano de cacao.

\subsection{Los estudios sobre cacao}

Al revisar la evidencia de estudios empíricos sobre el cacao, tanto a nivel internacional como nacional, se encontró que gran parte de los estudios se han centrado en temas como mejoramiento genético, identificación y caracterización de clones de cacao altamente productivos resistente a plagas y enfermedades, así como al análisis y mejoramiento de los procesos de transformación en la cadena de cacao.

Según Castellanos et al. (2007) las principales investigaciones a nivel mundial se centran en siete temáticas: mejoramiento genético, condiciones del cultivo, control fitosanitario, beneficio y fermentación, transformación primaria donde se tienen en cuenta la obtención de los primeros subproductos de la cadena (ej. licor de cacao, pasta de cacao y manteca de cacao), transformación secundaria donde se obtienen productos como chocolatería y finalmente investigaciones enfocadas a los productos finales y el consumidor final.

Así por ejemplo, Mejía F., y Arguello, O (2000) indican que el principal problema del cacao en Colombia es la baja calidad del grano, debido a la falta de estándares para los procesos. Asimismo, Castellanos et al (2007) identifican las limitaciones en la cadena productiva del cacao-chocolate y postulan las siguientes como las más importantes: asimetría en la oferta de soluciones tecnológicas en los diferentes eslabones de la cadena; bajos rendimientos de la producción de cacao en grano; baja productividad de las plantaciones, debido a la avanzada edad de los cultivos y a deficientes prácticas agronómicas; escaso apoyo e incentivos al pequeño productor y poca inversión por parte del Estado; escasez de mano de obra calificada, aunado a los altos costos de los jornales, divergencias en las metodologías para determinar costos de establecimiento y manejo de cultivo, así como en los valores de los mismos, 
según las zonas productoras; inestabilidad de los precios y falta de aplicación de las normas para la compra del cacao; poca organización por parte del gremio de cacaocultores con la consecuente dificultad para la asociatividad, y escasas prácticas administrativas de las unidades productivas, entre otros factores.

Sánchez et al. (2008) investigaron sobre mejoramiento de la poscosecha del cacao a partir del roadmapping encontrando que el manejo poscosecha del cacao en Colombia requiere desarrollo tecnológico, ya que las brechas tecnológicas existentes entre el manejo actual del proceso evidencian cierto rezago, lo que vislumbra el incipiente estado de desarrollo de la poscosecha de este producto frente a los referentes internacionales. Adicionalmente, se encontró que el sector cacaotero no está preparado para responder a las exigencias de calidad y de nuevos productos, como cacao orgánico certificado o productos nutracéuticos que requieren nichos particulares de mercado en crecimiento, ya que no posee la tecnología de poscosecha apropiada para garantizar el desarrollo controlado de las características organolépticas y de calidad exigidas. Por lo general, el proceso de poscosecha en países productores de cacao es caracterizado por la escasa tecnificación de los procesos y las arraigadas costumbres de los productores en el manejo del cultivo lo que en ocasiones conduce a la baja calidad del producto (Laven, 2005; Liendo, 2006). Muy pocos estudios han evaluado la calidad de vida de cacaoteros y el nivel de competitividad de los países productores.

\section{Metodología}

Para el análisis del sector del cacao se utilizó el modelo de las cinco fuerzas competitivas de Porter (2008), buscando alcanzar una caracterización económica dominante en la industria del cacao, determinar cuáles son las fuerzas competitivas operantes en ella, establecer los factores clave de éxito, identificar la rivalidad de la competencia, establecer qué tan atractiva es la industria cacaotera en términos de rentabilidad y brindar un marco para anticiparse a la competencia e influir en ella (y en la rentabilidad) en el largo plazo (Porter, 2008).

El modelo de Porter (2008) permite el empleo de un marco de referencia común para medir la presión competitiva en las industrias, teniendo en cuenta que cada industria es diferente. Este modelo puede explicarse mediante cinco factores: la rivalidad entre competidores, el ingreso potencial de nuevos competidores, el poder de negociación de los proveedores, el poder de negociación de los compradores y la amenaza de productos sustitutos (véase Figura 5.) 
Figura 5. Modelo de las cinco fuerzas de Porter

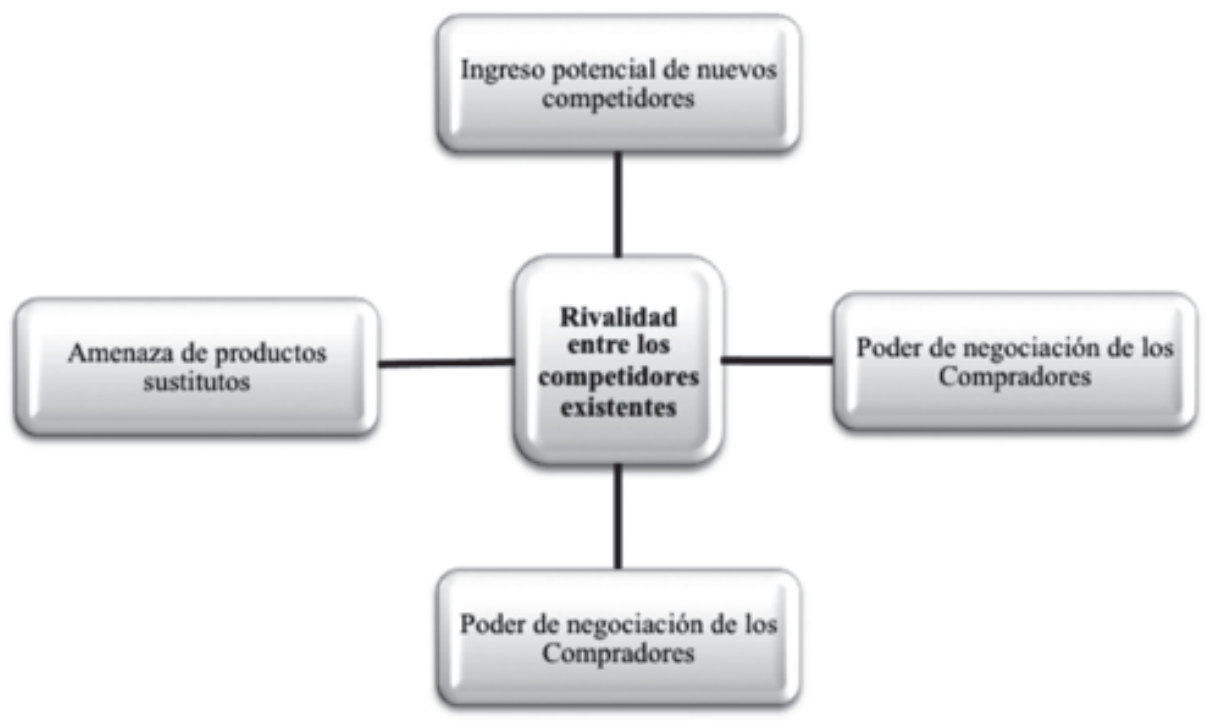

Fuente: Porter (2008).

\section{Datos y variables}

Para determinar el nivel de competitividad del sector de cacao, se escogió el Departamento de Santander que, como se mencionó anteriormente, es el Departamento que registra la mayor producción de cacao. La muestra de estudio fue de 265 productores de cacao ubicados en el municipio de San Vicente del Chucuri y 68 productores de cacao ubicados en el municipio del Carmen de Chucuri para un total de 333 productores. Se desarrolló y validó un instrumento de adquisición de datos primarios dirigida a los productores de cacao en los municipios seleccionados para el estudio.

El cuestionario utilizado permite recoger datos sobre diversos aspectos de los productores de cacao. Así, el instrumento recoge, por una parte, información personal como número de personas que forman el hogar y sobre su localización geográfica, nivel educativo, tipo de vivienda, entre otros. Por otra parte, se accede a información como economías de escala, lealtad del cliente, costo de cambio, requerimientos de capital, acceso a los canales de distribución, experiencia y efectos de aprendizaje, acceso a tecnología, política gubernamental, nivel de concentración, precio, localización, tipo de relación y calidad del producto, barreras emocionales, crecimiento de la industria, márgenes de la industria entre otros. 
Asimismo, estos datos han sido completados con otros a nivel de país extraídos de diversas fuentes secundarias como la Federación Nacional de Cacaoteros (Fedecacao), el Observatorio de Competitividad Agrocadenas Colombia, el Ministerio de Agricultura y Desarrollo Rural de Colombia MADR, el DANE y la base de datos Benchmark.

\section{Resultados}

\subsection{Amenazas de la entrada de competidores en la producción de cacao}

La Tabla 2 y la Figura 6 presentan algunas de las principales barreras a la entrada de nuevos competidores en los productores de cacao en Santander, donde se analizaron variables como economías de escala, lealtad del cliente, costo de cambio, requerimientos de capital, acceso a los canales de distribución, experiencia y efectos de aprendizaje, acceso a tecnología y política gubernamental.

Un aspecto importante a tener en cuenta en el análisis es la cultura de productores de cacao, ya que su interés sobre el cultivo depende principalmente del precio: cuando el precio es bajo y genera una mínima ganancia, se desatiende el cultivo y sólo se extrae la cosecha sin invertirle recursos (tiempo y dinero) para su mantenimiento (Martínez et al., 2005).

Al analizar las economías de escala, el nivel tecnológico y el requerimiento de capital, encontramos que el $47,1 \%$ de los productores no hacen uso de las economías de escala. El 57,4\% de los productores aseguran no tener tecnificados sus cultivos y el 39\% de los productores consideran que se necesita de un capital importante para producir. En la fase productiva de cacao se realizan labores como control de malezas y plagas, poda, deschupone, recolección de la cosecha, dado el bajo nivel tecnológico sólo un pequeño porcentaje de los productores realizan labores de fertilización, riego, drenaje y siembras. En general, los productores son conscientes de la importancia de hacer uso de las economías de escala para reducir sus costos y tecnificar sus cultivos, pero la falta de capital se los impide ya que en la región predomina el pequeño y mediano productor de economía campesina, con limitación de recursos.

Por otra parte, el desgaste económico de la inversión inicial y la tardanza en el retorno financiero son factores que tienen un peso relativo en el momento de tomar la decisión de producir cacao. En San Vicente del Chucurí y el Carmen de Chucurí el tamaño promedio de los cultivos de cacao es de seis hectáreas, éstas se hallan concentradas en un $67,1 \%$ en tamaños comprendidos entre tres y diez hectáreas, principalmente se trata de cultivos de tipo familiar para el sustento de los integrantes del núcleo familiar, pero no están concebidos como unidades agrícolas familiares.

Los productores consideran importante la lealtad del cliente y el costo de cambio de ellos es alto, en su mayoría venden su producción a las asociaciones, acopiadores 
y/o compraventas de cacao los cuales hacen el papel de intermediarios. El acceso a los canales de distribución, según los productores, es calificado entre regular y bueno, debido a que se consigue transporte regularmente y cualquier productor puede acceder a él. Sin embargo, observamos que el estado de las vías para sacar el producto de las fincas y de los pueblos es muy defectuoso, generalmente se ven afectadas por el invierno, existen zonas donde los productores los últimos años se han tenido que quedar con los productos en sus fincas, a falta de caminos por donde sacarlos. Las vías que se ven más afectadas son las que de San Vicente conducen a Barrancabermeja, el Carmen de Chucurí y Bucaramanga, además de los carreteables que comunican al municipio con sus diferentes veredas.

Finalmente, y a pesar de los esfuerzos del gobierno por favorecer a los cultivadores, evidenciamos un descontento generalizado de los productores encuestados en cuanto a la política gubernamental; el 86\% de ellos considera muy bajo el apoyo recibido del gobierno y los gremios, y no se identifican ni con las políticas ni con las estrategias desarrolladas por ellos.

Debido a lo anteriormente expuesto, podemos decir que el posicionamiento como nuevo productor de cacao en la región enfrenta bajas barreras de entrada, ya que los productores actuales no presentan factores diferenciales importantes y cualquier empresa con capital suficiente puede hacerles fuerte competencia.

Tabla 2. Amenaza de entrada de nuevos competidores

\begin{tabular}{|c|c|c|c|c|c|}
\hline \multirow{2}{*}{ Variables } & \multicolumn{5}{|c|}{ Intensidad } \\
\hline & Muy baja & Baja & Media & Alta & Muy Alta \\
\hline Economías de escala & & $\mathrm{X}$ & & & \\
\hline Lealtad del cliente (o "de marca") & & & & $\mathrm{X}$ & \\
\hline Costo de cambio & & & & $\mathrm{X}$ & \\
\hline Requerimientos de capital & & & & $\mathrm{X}$ & \\
\hline Acceso a canales de distribución & & & $\mathrm{X}$ & $\mathrm{X}$ & \\
\hline Acceso a la tecnología & $X$ & & & & \\
\hline Política gubernamental & $\mathrm{X}$ & & & & \\
\hline
\end{tabular}

Fuente: Elaboración de las autoras. 
Figura 6. Amenaza de entrada de nuevos competidores

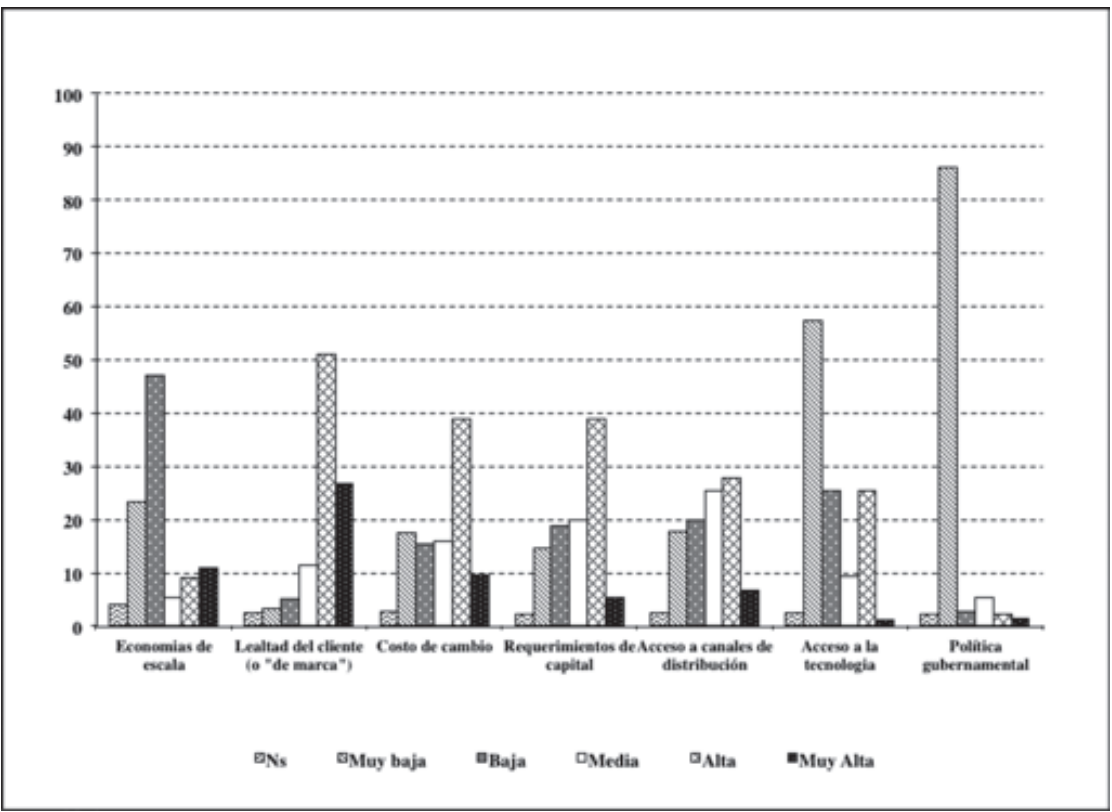

Fuente: Elaboración de las autoras

\subsection{Nivel de poder de negociación de los proveedores en producción de cacao}

La Tabla 3 y la Figura 7 presentan el nivel de poder de negociación de los proveedores de la producción de cacao de Santander.

La gran mayoría de los productores de cacao de la región compran sus insumos en el pueblo más cercano, lo que les da a los proveedores un amplio control sobre la obtención de las materias primas. El 44,1\% de los productores considera que el nivel de concentración de los proveedores el bastante alto y el $58 \%$ de los productores respondieron que los precios de los insumos son altos, en relación al servicio y la calidad ofrecida. Estos incrementos en los precios pueden deberse a los costos de los fletes para transportar la materia prima desde los pueblos y, en ocasiones, a la escasez de los insumos debido al deficiente estado de las vías. Aunque la relación con los proveedores no es muy cercana (media 58,3\%) el costo del cambio de proveedor si es bastante alto para el productor (58\%) puesto que muchos de los insumos se los proporciona a crédito. Los productores cuentan con poco capital disponible y se ven forzados a dejar la producción como prenda de garantía.

Con respecto a la facilidad de sustituir insumos vendidos por otros proveedores alternativos, hay que considerar el caso de cada insumo en particular. Asimismo, los proveedores en el pueblo son reducidos por lo que los productores no pueden 
darse el lujo de contar con un amplio abanico de proveedores alternativos. Es factible que la mayoría de los productores de cacao no estén bien informados sobre el funcionamiento del mercado del producto brindado por el proveedor, lo que los pone en desigualdad de condiciones frente a los proveedores. En general, se encontró que los proveedores tienen alto poder de negociación, tanto en precio como en el servicio y la calidad ofrecida.

Tabla 3. Relación con los Proveedores

\begin{tabular}{|c|c|c|c|c|c|}
\hline \multirow[b]{2}{*}{ Variables } & \multicolumn{5}{|c|}{ Intensidad } \\
\hline & Muy baja & Baja & Media & Alta & $\begin{array}{l}\text { Muy } \\
\text { Alta }\end{array}$ \\
\hline Concentración de los proveedores & & & & $\mathrm{X}$ & \\
\hline Precio de los insumos & & & & $\mathrm{X}$ & \\
\hline Localización & & & & $\mathrm{X}$ & \\
\hline Relación & & & $\mathrm{X}$ & & \\
\hline Peligro de integración hacia delante & & & & $\mathrm{X}$ & \\
\hline Costo de cambio de proveedor & & & & $\mathrm{X}$ & \\
\hline Calidad del producto & & & $\mathrm{X}$ & $\mathrm{X}$ & \\
\hline
\end{tabular}

Fuente: Elaboración de las autoras.

Figura 7. Relación con los proveedores

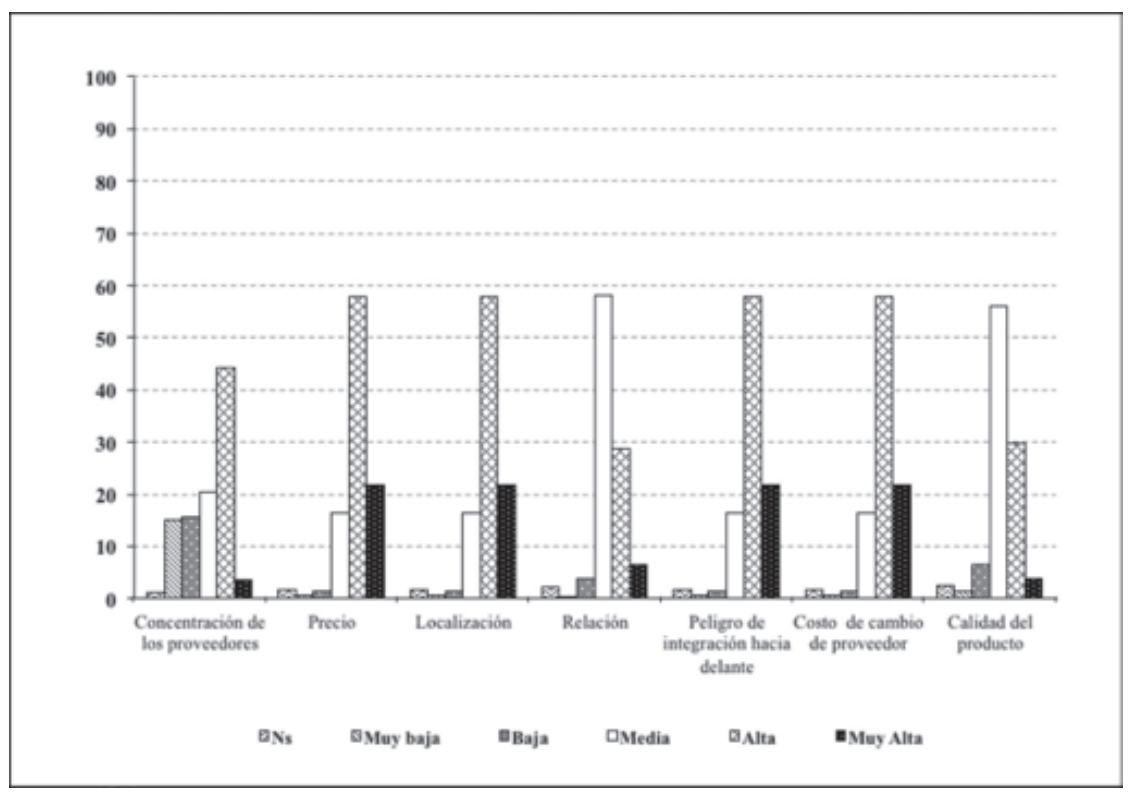

Fuente: Elaboración de las autoras. 


\subsection{Nivel de poder de negociación de los clientes en la producción de cacao}

El poder negociador de los clientes, junto con el poder de negociación de los proveedores, afecta directamente los resultados obtenidos por los productores del sector cacaotero, debido a que de ello depende la forma de reparto del margen total generado a lo largo de la cadena de valor en la que participan todos ellos. Cuanto mayor sea el poder de los clientes, mayor será su capacidad de negociación con el productor de cacao, exigiendo menores precios y erosionando los beneficios del sector productor.

La Tabla 4 y la Figura 8 presentan el nivel de poder de negociación de los clientes de la industria de cacao de Santander. El 51\% de los productores consideran que los clientes tienen un costo bajo por cambiar de productor. Asimismo, los productores venden su cosecha generalmente al mismo cliente por lo cual lo consideran muy importante.

Como hemos comentado anteriormente el poder de negociación y la definición de los precios está determinado por el lado de la demanda del producto, es decir, el precio está determinado básicamente por la industria transformadora. Los precios pagados por los comisionistas responden a los establecidos por las grandes industrias, las cuales han determinado vincular el precio interno con el precio del mercado internacional, debido a esto el precio interno corresponde aproximadamente al $88 \%$ - 90\% del precio en la bolsa de Nueva York (Espinal et al., 2005).

Por otra parte, el 52\% de los productores de cacao de San Vicente del Chucurí consideran que es alto el riesgo de que sus clientes se integren verticalmente hacía atrás. La industria chocolatera en Colombia presenta un importante grado de integración con el cultivo del cacao. Así por ejemplo, la granja Lucker - centro cacaotero creado desde hace 32 años - realiza gestión en el área de capacitación. Aproximadamente 26.990 personas han recibido instrucción en La Granja a través de 396 cursos de cacao, giras, reuniones y eventos técnicos de cobertura internacional. De la misma forma, la Empresa Nacional De Chocolates ha creado programas integrales de apoyo y fomento agrícola con el objetivo de fortalecer la cadena de suministro de la empresa y asegurar la compra de la cosecha. En resumen, el poder de negociación de los clientes es muy alto debido a las características duopsónicas de la industria. 
Tabla 4. Relación con los Clientes

\begin{tabular}{lccccc}
\hline \multirow{2}{*}{ Variables } & \multicolumn{4}{c}{ Intensidad } \\
\cline { 2 - 5 } & Muy baja & Baja & Media & Alta & Muy Alta \\
\hline Costo del cliente por cambiar de productor & $\mathrm{X}$ & & \\
Clientes importantes sobre el total & & $\mathrm{X}$ & $\mathrm{X}$ \\
\hline Amenaza de integración hacia atrás & & & $\mathrm{X}$ \\
\hline
\end{tabular}

Fuente: Elaboración de las autoras.

Figura 8. Relación con los Clientes

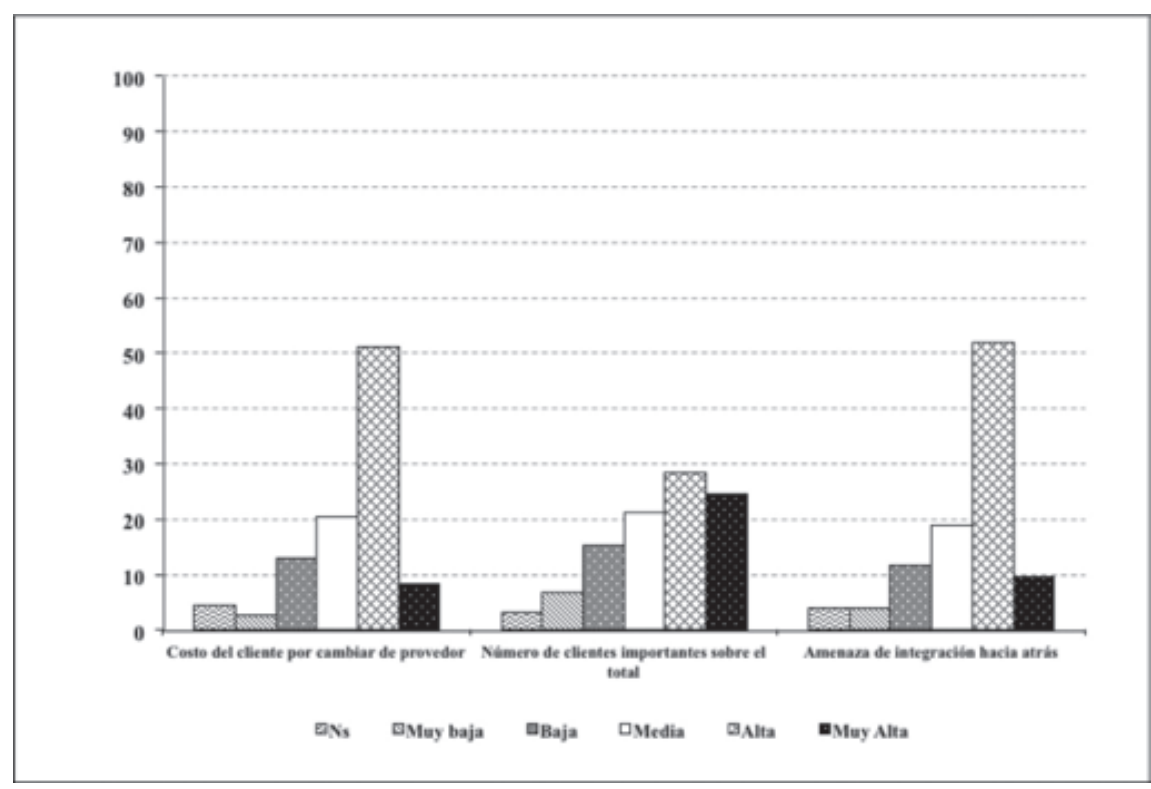

Fuente: Elaboración de las autoras.

\subsection{Nivel de rivalidad entre los productores de cacao}

La Tabla 5 y la Figura 9 presentan los principales resultados para la rivalidad entre los productores de cacao de la región. El 75\% de los productores encuestados considera que el principal motivo de rivalidad es la existencia de muchos productores de cacao en la zona en relación al crecimiento de la industria, el cual consideran entre bajo y medio (34,8\% y 35,7\%) aunado al limitado margen de beneficio, el cual califican de bajo. De lo anterior se deduce que algunos de los factores más importantes 
que definen esta rivalidad son las barreras emocionales de los productores, que se considera se presentan en un grado alto (39.6\%).

La gran mayoría de productores de cacao son dueños y/o arrendados de las fincas y su cosecha es el único medio de subsistencia. De la misma manera, es una labor que se ha venido realizando de generación en generación. En general, la rivalidad de los productores de cacao es alta debido a la gran cantidad de agricultores de la zona y a las barreras emocionales. No obstante, esta rivalidad no incide con mayor fuerza en el ámbito de la innovación, la tecnología y los precios.

Tabla 5. Rivalidad entre competidores

\begin{tabular}{cccccc}
\hline \multirow{2}{*}{ Variables } & \multicolumn{5}{c}{ Intensidad } \\
\cline { 2 - 5 } & Muy baja & Baja & Media & Alta & Muy Alta \\
\hline Número de competidores & & & & X \\
\hline Barreras emocionales & & & & \\
\hline Crecimiento de la industria & & $\mathrm{X}$ & $\mathrm{X}$ & \\
\hline Márgenes de la industria & $\mathrm{X}$ & $\mathrm{X}$ & & \\
\hline
\end{tabular}

Fuente: Elaboración de las autoras.

Figura 9. Rivalidad entre competidores

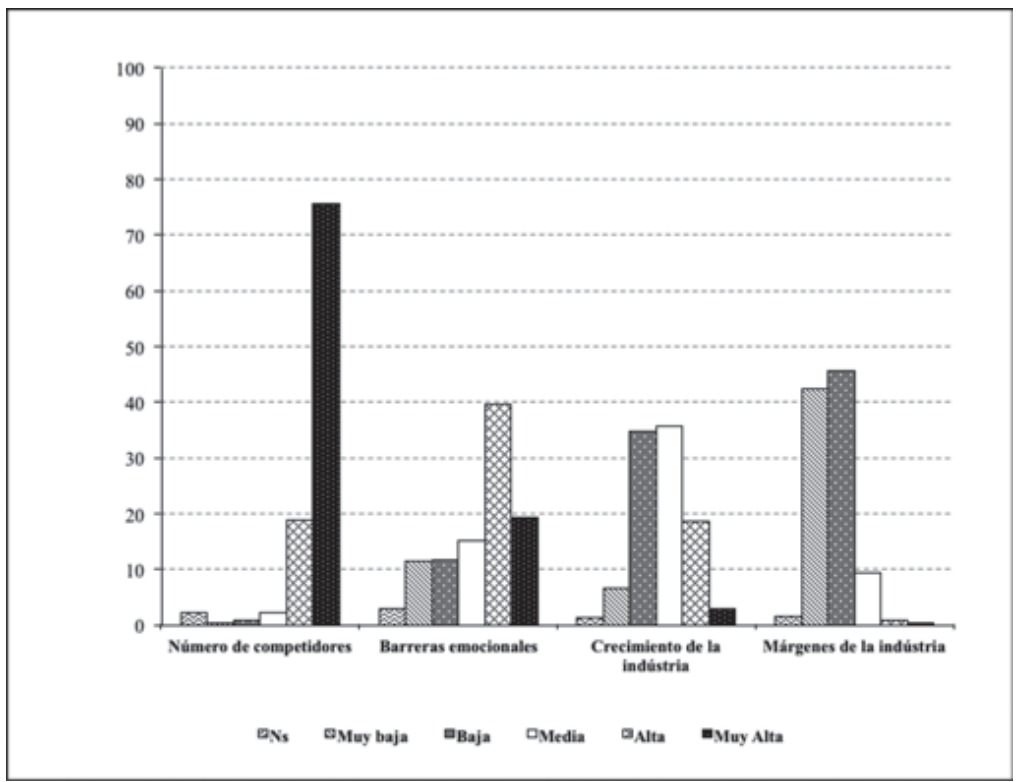

Fuente: Elaboración de las autoras. 


\subsection{La presión de productos sustitutos}

La cocoa es uno de los productos sustitutos que proviene de otros países, es de inferior calidad y se está utilizando en procesos industriales en la pequeña industria de chocolate de mesa, panaderías, chocolaterías, confiterías y galletería. En el año2010, se importaron en Colombia 3.500 toneladas de este producto, y en año 2011 se importaron 4.500 toneladas, con cero aranceles y a precios módicos. Según los productores, ya no se necesita un horno para tostar los granos ni un molino para molerlos, la cocoa llega en polvo y se utiliza para todas las labores donde antes estaba el cacao.

De igual forma, la industria y las empresas proveedoras de ingredientes se han venido esforzando por encontrar una solución a la inestabilidad del cacao y desarrollaron una alternativa para el reemplazo de los distintos perfiles de cacao: los "sustitutos de cacao", así por ejemplo NeoCao es la línea de sustitutos de cacao desarrollada y comercializada por SAPORITI S.A. Este tipo de producto se caracteriza por ser una solución integral, ya que aporta al mismo tiempo el sabor y el color característico del cacao. En general, se puede reemplazar hasta un $100 \%$ del cacao, aunque se sugiere, desde el punto de vista tecnológico, sustituir hasta un 50\% del cacao de acuerdo a la aplicación.

\section{Conclusiones}

Por todo lo anterior, y de acuerdo a los resultados alcanzados con la intensidad de cada una de las fuerzas, se encontró que la producción de cacao durante los últimos años se ha convertido en una actividad productiva poco atractiva para la inversión, y que los grandes márgenes de rentabilidad se los están quedando las empresas transformadoras de la cadena productiva. Es evidente que los productores de cacao vienen pasando por una fuerte crisis debido a los bajos precios que pagan por su producción, razón por la cual los productores piden la compra de la cosecha por parte del Gobierno Nacional a un precio justo.

A pesar de los grandes esfuerzos del gobierno nacional y regional, los productores muestran un descontento generalizado con los gremios y programas del gobierno que buscan mejorar la productividad y competitividad del sector debido, por una parte, la mala planificación de cada uno de los programas, a la agendas paralelas que se llevan, a la promoción de los programas sin llegar a un empoderamiento de los diferentes proyectos y en general, a la gran cantidad de proyectos inconexos, sin articulación entre ellos.

Asimismo, los entes financiadores presentan muchas exigencias al momento de prestar capital en este tipo de cultivos, los seguros agrícolas no cubren todos los riesgos de una producción agropecuaria. Por tanto, los productores se ven obligados a realizar préstamos paralelos pagando intereses muy altos y comprometiendo su producción. 
En esta zona, el nivel de asociatividad es muy incipiente, tan solo el 30\% de los productores están asociados a 16 cooperativas y éstas no cumplen a cabalidad los objetivos para los que fueron creadas. Se debe fomentar una mayor organización y la asociatividad entre productores y proporcionarles el apoyo requerido para garantizar un mayor empoderamiento, en términos administración de la producción e implementación y uso tecnológico que les permitan ser más productivos y competitivos, con el fin de ubicarlos con mayor poder negociador en la cadena productiva.

\section{Referencias}

Asamoah, D., y Annan, J. (2012). Analysis of Ghana's cocoa value chain towards services and standards for sta keholders. International Journal of Services and Standards, 8(2), 116-132.

Bueno, T. (2004). Diagnóstico de la cadena productiva del cacao-chocolate en el departamento de Arauca [Cd Room].

Castellanos, Ó. F., Torres, L. M., Fonseca, S. L., Montañez, V. M., y Sánchez, A. (2007). Agenda prospectiva de investigación y desarrollo tecnológico para la cadena productiva de cacao-chocolate en Colombia. Proyecto Transición de la Agricultura. Ministerio de Agricultura y Desarrollo Rural y Biogestión, Universidad Nacional de Colombia, Bogotá

CORPOICA Y FEDERACIÓN NACIONAL DE CACAOTEROS (2009) Caracterización y zonificación de áreas potenciales para el cultivo del cacao en Colombia. Dirección de cadenas productivas. Cadena Cacao-Chocolate. 36 p.

Espinal, C., Martinez, H., y Beltrán, L. (2005). La industria de chocolates en Colombia. Documento de trabajo Retrieved 76, Disponible en: www.agrocadenas.gov.co/ documentos/anuario2005/Capitulo3_Chocolates.pdf

FAO. (2004). Perspectivas a plazo medio de los productos básicos agrícolas. FAO Commodities and Trade Technical Paper, 192 pp

Gamboa, C., Zuluaga, S., y Rubiano, E. (2007). Características y funcionamiento del mercado de cacao y sus derivados en colombia. Proyecto de investigación para la Compañía Nacional de Chocolates S.A. FEDESARROLLO

González, V. (2005). Cacao en Mexico: Competitividad y medio ambiente con alianzas INIFAP e IPRC para USAID. México. Informe técnico Publicaciones USAID 
ICCO. (2007). Junta Consultiva Sobre la Economía Cacaotera Mundial. Economía Cacaotera Sostenible: Un enfoque amplio y participativo. ICCO: International Cocoa Organization. Disponible en http://www.icco.org/about-us/icco-news. html Consultado el 12-10-2011

ICCO. (2012). The world cocoa economy: past and present international cocoa organization. EX/146/7. London UK. Disponible en: http://www.icco.org/aboutus/international-cocoa-agreements/cat_view/30-related-documents/45-statisticsother-statistics.html

INFOCOMM. (2007). Información de mercado sobre productos básicos. Cacao. Disponible en: http://www.unctad.org/infocomm/espagnol/cacao/cadena.htm Consultado el 12-12-2012

Martínez, H. J., Espinal G., C. F., y Ortiz Hermida, L. (2005). La cadena del cacao en Colombia: una mirada global de su estructura y dinámica 1991-2005 (Vol. Documento de trabajo 58): Ministerio de Agricultura y Desarrollo Rural MINAGRICULTURA.

Mojica, A y Paredes, J (2006). Caracteristicas del cultivo de cacao en Santander. Banco de la Republica de Colombia.

Ofori-Bah, A., y Asafu-Adjaye, J. (2011). Scope economies and technical efficiency of cocoa agroforesty systems in Ghana. Ecological Economics, 70(8), 1508-1518.

Porter, M. (2008). Las cinco fuerzas competitivas que le dan forma a la estrategia. Harvard Business Review, 86(1), 58-77.

ProExport. (2012). Cacao Colombiano fino y de aroma. Consultado en junio 3 de 2013. Diponible en:http://www.inviertaencolombia.com.co/images/Perfil\%20 Cacao\%202012.pdf 